This item was submitted to Loughborough's Research Repository by the author.

Items in Figshare are protected by copyright, with all rights reserved, unless otherwise indicated.

\title{
Delayed creation of entanglement in superconducting qubits interacting with
} a microwave field

\section{PLEASE CITE THE PUBLISHED VERSION}

http://dx.doi.org/10.1140/epjb/e2010-00056-y

\section{PUBLISHER}

(c) Springer-Verlag

\section{VERSION}

AM (Accepted Manuscript)

\section{LICENCE}

CC BY-NC-ND 4.0

\section{REPOSITORY RECORD}

Abdel-Aty, M., and Mark J. Everitt. 2019. "Delayed Creation of Entanglement in Superconducting Qubits Interacting with a Microwave Field”. figshare. https://hdl.handle.net/2134/12095. 
This item was submitted to Loughborough's Institutional Repository (https://dspace.lboro.ac.uk/) by the author and is made available under the following Creative Commons Licence conditions.

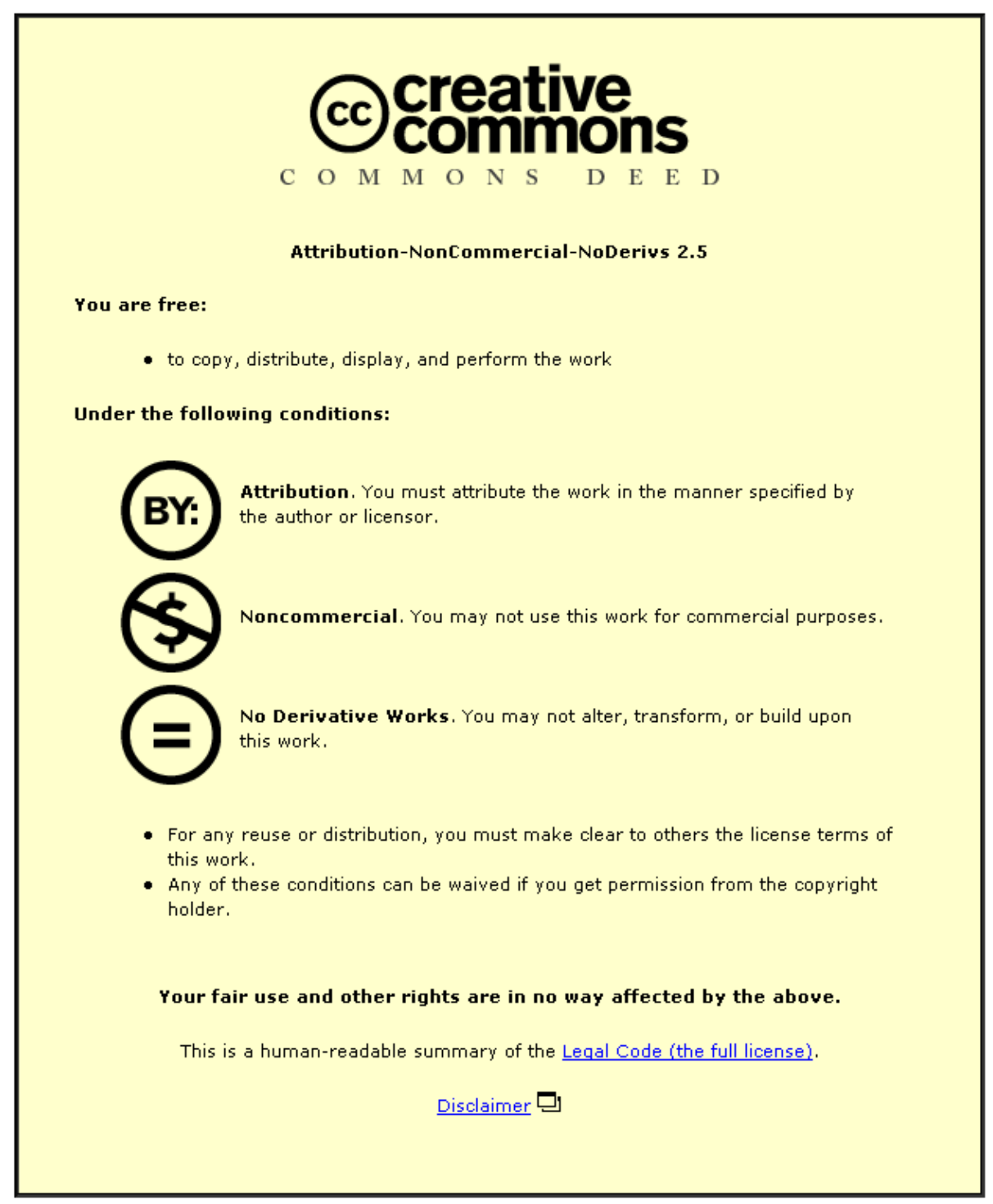

For the full text of this licence, please go to: http://creativecommons.org/licenses/by-nc-nd/2.5/ 


\title{
Delayed creation of entanglement in superconducting qubits interacting with a microwave field
}

\author{
M. Abdel-Aty ${ }^{*}$ \\ Mathematics Department, College of Science, Bahrain University, Bahrain \\ Laboratoire Collisions Agrgats Ractivit - UMR5589, \\ Universit Paul Sabatier, 31062 Toulouse Cedex 09, France \\ Mark Everitt \\ Centre for Theoretical Physics, The British University, \\ El Sherouk City, Postal No. 11837, P.O. Box 43, Egypt \\ Department of Physics, Loughborough University, Leicestershire, LE11 3TU, UK.
}

(Dated: March 2, 2013)

\begin{abstract}
We explore the role played by the intrinsic decoherence in superconducting charge qubits in the presence of a microwave field applied as a magnetic flux. We study how the delayed creation of entanglement, which is opposite to the sudden death of entanglement, can be induced. We compute the time evolution of the population inversion, total correlation and entanglement, taking into account the junction mixed state and dissipation of the cavity field. We show that although decoherence destroys the correlation of the junction and field, information of the initial state may be obtained via quasi-probability distribution functions.
\end{abstract}

\section{INTRODUCTION}

Over the last decade, superconducting qubits have gained substantial interest as devices for application in quantum information processing ${ }^{1,2}$. Here, Josephson qubits are recognized as being among the most promising devices to implement solid state quantum computation ${ }^{3}$. The manipulation of quantum states in individual and coupled qubits (Cooper-pair box) has been demonstrated experimentally in [4] and the behavior of charge oscillations in superconducting Cooper pair boxes weakly interacting with an environment has been discussed in [5]. Superconducting circuits can behave like atoms and test quantum mechanics at macroscopic scales and be used to conduct atomic-physics experiments on a silicon chip 1 . Furthermore, the quantum dynamics of a Cooper-pair box with a superconducting loop in the presence of a nonclassical microwave field have been investigated in [6] .

In order to utilize superconducting circuits as a resource for quantum information processing the problem of engineering entangled states in coupled systems must be addressed. One of the most important problems under consideration is how to make a long-lived and easily monitored entangled states within existing experimental set-ups ${ }^{7}$. At present, one of the main obstacles in the development of a larger-scale solid state quantum logic circuit is decoherence. It is therefore important to develop strategies to minimize the effects of decoherence on the dynamics of the qubit systems 8,11 . Much of the work that has been done in this field focuses on decoherence due to coupling to environmental degrees of freedom. However, there exists another intriguing possible source of fundamental decoherence that was proposed by Milburn in [9]. This arises if, instead of being a continuum, time is considered to progress with some minimum, but finite, increment. Hence, the dynamics is not governed by a single unitary evolution operator but rather some stochastic se- quence of identical incremental unitary transformations. Taking this into account results in an equation of motion resembling that of master equations for Markovian open quantum systems. In other words, introducing a descritization of time results in a fundamental decoherence that affects any quantum system. It is therefore natural to ask what limits such intrinsic decoherence would place on applications in quantum information processing. Here we build on other work in this field ${ }^{32}$ and study the delayed creation of entanglement in superconducting qubits interacting with a microwave field.

The system that we study in this work closely resembles that of a qubit coupled to a quantum field mode which can be effectively represented using the JaynesCummings model ${ }^{21,22}$. A recent study has indicated that this system can be used to understand the quantum to classical transition of a field mode when a suitable model of environmental decoherence is introduced 10 . Hence, by observing the behaviour of the dynamics of the atomic inversion, the qubit can be used as a probe to estimate the amount of environmental dechorence to which the field is subjected. Milburn's model is slightly different in that decoherence arises because time is taken to increment, discontinuously, over short intervals. As the system does not evolve continuously, but under a stochastic sequence of identical unitary transformations, the associated decoherence effects the qubit and field equally. Nevertheless, it is our suggestion that systems that exhibit collapse and revival phenomena will provide a possible platform from which to test Milburn's proposal. The discontinuous evolution that is central to this model is independent of the system under consideration. Any test, or estimate of the time increment, should be obtained from a number of physically different sample systems which exhibit similar dynamical properties. Hence, collapse and revival forms good basis for testing intrinsic decoherence as this behaviour is seen in a number of different physical systems 
and is well understood. In this paper our objective is to demonstrate proof of principal and show the effect that intrinsic decoherence might have on a realistic system, a cooper pair box coupled to a non-classical field (we have used experimental parameters from [44]). We note that due to the existence of other decohering effects it may not be practically possible to establish the validity (or not) of intrinsic decoherence if the time increments are too small. Even so, we believe that studying collapse and revival phenomena with carefully controlled environments will provide an upper bound to the size of the interval.

Specifically, we study the junction-field dynamics and their associated entanglement properties. We demonstrate a protocol for entanglement engineering and characterization by studying a special type of superconducting charge qubit, namely a single Cooper-pair box coupled to a microwave field applied as a magnetic flux. We pay careful attention to the crucial difference between the strong or weak field regimes effect on the total correlation of the junction-field system. In order to complete our study from the perspective of a phase space approach we discussed the Wigner quasi-probability distribution. The present work is motivated by experimental results on Josephson junction and normal metal flux qubits coupled to the environment $\underline{\underline{16}}$. We note that some theoretical discussions and analysis of special cases of the problem at hand were given in Refs. 6, 17, 18 and experimental results were predicted in Ref. 16.

The organization of this paper is as follows: in section 2 we introduce the model and formulate the master equation and present its time-dependent analytical solution. In section 3 , as an application, we employ the analytical results obtained in section 2 to discuss the $\mathrm{AC}$ Josephson effect and entanglement for different values of the intrinsic decoherence. In section 4 , we focus on the Wigner function which corresponds to the final state of the charge-qubit system. Finally, we summarize the results in section 5 .

\section{THE MODEL}

We consider a superconducting box with a lowcapacitance Josephson junction with the capacitance $C_{J}$ and Josephson energy $E_{J}$, biased by a voltage source $V_{g}$ through a gate capacitance $C_{g}$ which is externally controlled and used to induce offset charges on the island. The schematic picture of this single-qubit structure is shown in figure 1. The total Hamiltonian of the system can then be written as 19

$\hat{H}=\frac{\left(Q-C_{g} V_{g}\right)^{2} e}{C_{g}+2 C_{J}}-2 E_{J 0} \cos \phi \cos \left(\frac{\pi \Phi}{\Phi_{0}}\right)+\hbar \omega\left(a^{\dagger} a+\frac{1}{2}\right)$,

where $a^{\dagger}$ and $a$ are, respectively, the creation and annihilation operator of the cavity mode. In this structure, the superconducting island with Cooper-pair charge $Q=2 N e$ is coupled to a segment of a superconducting ring via two Josephson junctions, where $e$ is

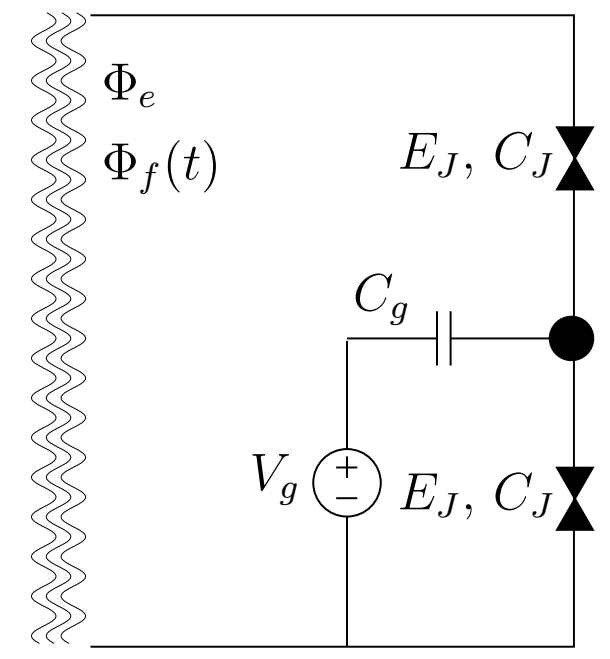

FIG. 1: Schematic picture of the Cooper-pair box. The filled circle denotes a superconducting island, the Cooper-pair box, which is biased by a voltage $V_{g}$ through the gate capacitance $C_{g}$ and coupled to the bulk superconductors by two identical small Josephson junctions. The two Josephson junctions have capacitance $C_{J}$ and Josephson energy $E_{J}$. The total flux is the summation of the static magnetic flux $\Phi_{e}$, and microwavefield-induced flux $\Phi_{f}(t)$ that applied via the superconductingquantum-interference-device loop.

the electron charge and $N$ is the number of Cooperpairs. We denote by $\phi=0.5\left(\phi_{1}+\phi_{2}\right)$ the phase difference across the junction. The gauge-invariant phase drops $\phi_{1}$ and $\phi_{2}$ across the junctions are related to the total flux $\Phi$ through the superconducting-quantuminterference-device (SQUID) loop by the constraint $\phi_{2}-$ $\phi_{1}=2 \pi \Phi / \Phi_{0}$, where $\Phi_{0}=h / 2 e$ is the flux quantum. Here, $E_{J}=2 E_{J 0} \cos \phi \cos \left(\pi \Phi / \Phi_{0}\right)$ and self-inductance effects on the single-qubit structure is ignored.

When a nonclassical microwave field is applied, the total flux is a quantum variable $\Phi=\Phi_{e}+\Phi_{f}(t)$, where $\Phi_{f}$ is the microwave-field-induced flux. If we consider a planar cavity and the SQUID loop of the charge qubit is perpendicular to the cavity mirrors, the vector potential of the nonclassical microwave field can be written as $A(r)=\left|u_{\lambda}(r)\right|\left(a^{\dagger}+a\right) A$, where a single-qubit structure is embedded in the microwave cavity with only a single photon mode $\lambda$. Thus, the flux $\Phi_{f}$ can be written as $\Phi_{f}=\left|\Phi_{\lambda}\right|\left(a^{\dagger}+a\right)$, where $\Phi_{\lambda}=\oint u_{\lambda} . d l$, where the contour integration is over the interior of the SQUID loop. We shift the gate voltage $V_{g}$ (and/or vary $\Phi_{e}$ ) to bring the single-qubit system into resonance with $k$ photons: $E \approx k \hbar \omega_{\lambda}, k=1,2,3, \ldots$ It is to be noted that the charge states are not the eigenstates of the Hamiltonian (2), so that the Hamiltonian can be diagonalized yielding the following two charge states $|e\rangle=\cos \xi|1\rangle-\sin \xi|0\rangle$ and $|g\rangle=\sin \xi|1\rangle+\cos \xi|0\rangle$ with $\xi=\frac{1}{2} \tan ^{-1}\left(E_{J} / 2 \varepsilon\right)$, where $\varepsilon=2 E_{c}\left(C_{g} V_{g} e^{-1}-(2 n+1)\right)$. Employing these eigenstates to represent the qubit, expanding the functions $\cos \left(\pi \Phi_{e} / \Phi_{0}\right)$ and using the rotating wave approximation, 
one can derive the total Hamiltonian of the system $\operatorname{as}^{20}$.

$$
\begin{aligned}
\hat{H}=\hbar \omega\left(n+\frac{1}{2}\right)+ & \left\{\frac{1}{2} E-E_{J 0} \sin (2 \xi) \cos \left(\frac{\pi \Phi_{e}}{\Phi_{0}}\right) f(n)\right\} \sigma_{z}^{\gamma^{-1}}, \text { by } \\
& +\cos (2 \xi) E_{J_{0}}\left\{a^{k} g_{(k)}(n) \sigma_{+}+a^{\dagger k} g_{(k)}^{*}(n) \sigma_{-}(2)\right.
\end{aligned}
$$

$$
\frac{d}{d t} \hat{\rho}(t)=-\frac{i}{\hbar}[\hat{H}, \hat{\rho}]-\frac{\gamma}{2 \hbar^{2}}[\hat{H},[\hat{H}, \hat{\rho}]]
$$

We denote by $\sigma_{ \pm}$and $\sigma_{z}$ the Pauli matrices in the pseudo-spin basis and $g_{k}(n)$ represents the $k$-photonmediated coupling between the charge qubit and the microwave field, and are given by $g_{(1)}(n)=$ $\sin \left(\frac{\pi \Phi_{e}}{\Phi_{0}}\right)\left(\phi-\frac{1}{2 !} \phi^{3} n+\frac{1}{4 !} \phi^{5}\left(2 n^{2}+1\right)-\ldots ..\right), g_{(2)}(n)=$ $\cos \left(\frac{\pi \Phi_{e}}{\Phi_{0}}\right)\left(\frac{1}{2 !} \phi^{2}-\frac{2}{4 !} \phi^{4}(2 n-1)+\ldots . ., g_{(3)}(n)\right)=$ $\sin \left(\frac{\pi \Phi_{e}}{\Phi_{0}}\right)\left(-\frac{1}{3 !} \phi^{3}+\frac{5}{5 !} \phi^{5}(n-1)-\ldots ..\right)$, etc., $f(n)=$ $\frac{1}{2 !} \phi^{2}(2 n+1)-\frac{3}{4 !} \phi^{4}\left(2 n^{2}+2 n+1\right)+\ldots ., n=a^{\dagger} a$ and $\phi=\pi \frac{\left|\Phi_{\lambda}\right|}{\Phi_{0}}$.

We note that this model is similar to that of the Jaynes-Cummings Hamiltonian ${ }^{21,22}$, the difference arising in the coupling and intensity dependent term $E_{J 0} \sin (2 \xi) \cos \left(\frac{\pi \Phi_{e}}{\Phi_{0}}\right) f(n)$. It is well known that the Jaynes-Cummings model exhibits collapse and revival phenomena. That is, although the qubit initially exhibits Rabi like oscillations these apparently disappear and then subsequently revive $e^{22,23,24,25}$. This phenomena is well understood both theoretically $26,27,28$ and experimentally ${ }^{30.40}$. Hence, we might expect this system to behave in a similar fashion. As we will see, this is indeed the case.

\section{A. Solution}

The intrinsic decoherence approach ${ }^{9,31,33,34,35}$, is based on the assumption that on sufficiently short-time steps the system does not evolve continuously under unitary evolution but rather in a stochastic sequence of identical unitary transformations. Under the Markovian where $\gamma$ is the intrinsic decoherence parameter. The first term on the right-hand side of equation (3) generates a coherent unitary evolution of the density matrix, while the second term represents the decoherence effect on the system and generates an incoherent dynamics of the qubits system. In order to obtain an exact solution for the density operator $\hat{\rho}(t)$ of the master equation (3), three auxiliary superoperators $J, S$ and $L$ can be introduced $^{9,31}$ as $J \hat{\rho}(t)=\gamma \hat{H} \hat{\rho}(t) \hat{H}, S \hat{\rho}(t)=-i[\hat{H}, \hat{\rho}(t)]$ and $L \hat{\rho}(t)=-(\gamma / 2)\left(\hat{H}^{2} \hat{\rho}(t)+\hat{\rho}(t) \hat{H}^{2}\right)$.

Unlike state vectors, the density matrix can represent statistical mixtures of states. Hence, intrinsic decoherence implies that pure states may evolve into mixed states. It is intriguing to ask a related question; if some component of a system is initially in a mixed state is it possible, in the presence of intrinsic decoherence, to use this state to develop resources for quantum information processing. In order to prepare such an initial state we might consider the deliberate, and temporary, application of an appropriate decohering environment. Alternatively, it may be possible to apply some form of guidance law $\underline{37}$ to attain the desired state within the Bloch sphere. Here, we suppose that the initial state of the Cooper pair box is given by $\rho_{A}(0)=\varsigma_{1}|e\rangle\left\langle e\left|+\varsigma_{2}\right| g\right\rangle\langle g|$, where $\varsigma_{i} \geq 0$, and $\varsigma_{1}+\varsigma_{2}=1$. In addition, we suppose that the initial state of the field is given by $\rho_{F}(0)=|\varpi\rangle\langle\varpi|$, where $|\varpi\rangle=\sum_{n=0}^{\infty} b_{n}|n\rangle$, and $b_{n}^{2}=|\langle\varpi \mid n\rangle|^{2}$ being the probability distribution of photon number for the initial state. It is then straightforward to write down the formal solution of the master equation (3) as follows

$$
\begin{aligned}
\hat{\rho}(t) & =\exp (J t) \exp (S t) \exp (L t) \hat{\rho}(0) \\
& =\sum_{k=0}^{\infty}\left\{\varsigma_{1} \hat{M}_{k}(t)|e, \varpi\rangle\left\langle\varpi, e\left|\hat{M}_{k}^{\dagger}(t)+\varsigma_{2} \hat{M}_{k}(t)\right| g, \varpi\right\rangle\langle\varpi, g| \hat{M}_{k}^{\dagger}(t)\right\},
\end{aligned}
$$

where $\hat{\rho}(0)$ is the density operator of the initial state of the system and

$$
\hat{M}_{k}=\frac{(\gamma t)^{k / 2}}{\sqrt{k !}} \hat{H}^{k} \exp (-i \hat{H} t) \exp \left(-\frac{\gamma t}{2} \hat{H}^{2}\right) .
$$

$$
\sum_{k=0}^{\infty} \hat{M}_{k}(t) \hat{M}_{k}^{\dagger}(t)=\hat{I} \text { for all } t
$$

The so-called Kraus operators $\hat{M}_{k}$ satisfy 


\section{DYNAMICS}

\section{A. AC Josephson effect}

The AC Josephson effect can be used to observe collapse and revival in a condensed matter system. AC Josephson effect involve interaction of the photons with a junction which behaves like an atom undergoing transition between the quantum states of each side of the junction as it adsorbs and emits radiation. To see this phenomenon, we calculate the the time dependence of the populations inversion $\left\langle\hat{\sigma}_{z}(t)\right\rangle$ for different values of the decoherence parameter $\gamma$.

In Fig. 2, we plot the populations inversion and tangle as a function of the scaled time $\lambda t$ where $\lambda=\sqrt{e^{2} \omega / \hbar C_{F}}$. In this figure, we use the generalized mixed state form of the tangle ${ }^{36} S_{a}=2 \min _{S_{i}} \sum_{i}\left(1-\operatorname{Tr}\left[\left(\rho_{a}^{(i)}\right)^{2}\right]\right)$, where $\rho_{a}^{(i)}$ is the marginal state for the $i^{\text {th }}$ term in the ensemble decomposition and $S_{i}$ is a convex combination of the pure states. In the pure state case this definition will be reduced to the usual from of the tangle $S_{a}=2\left(1-\operatorname{Tr} \rho_{a}^{2}\right)$. Here we consider the single photon process and $\Phi_{e}=\Phi_{0} / 2$ for all Josephson charge qubits, and the gate voltage is adjusted to have the qubit on resonance with the cavity mode. It is interesting to show collapse and revival of the populations inversion as the system evolves, from an initial coherent state, for the microwave field. The interesting feature of the present case is that the general state without decoherence effect is host to entanglement between the junction and the field and this provides an opportunity to study the dynamics of such entanglement. To throw further light on the matter we also show, in Fig. 2, the time evolution of the mixed state tangle calculated from $\hat{\rho}(t)$ for the mixed initial state. Evidently, just as the occupation of the initial qubit states collapses and revives, so does the tangle. In close analogy with the Jaynes-Cummings model, the tangle remains near zero for long periods between revivals. The deterioration of revivals may be seen for $\gamma / \lambda=0.01$ where the amplitude of oscillations is smaller than in the case for larger $\gamma / \lambda$ where the revivales do not occur. Once the initial state of the field is considered to be a thermal state, the collapse-revival structure disappears. This initial atomic inversion collapses to the mid-level and does not oscillate for some period of time, which is followed by a quasichaotic behavior (in agreement with the standard two-level system ${ }^{38}$ ).

It is evident that the effect of the mixed state parameter $\theta=\pi / 3$ leads to decreasing of $\left\langle\hat{\sigma}_{z}(t)\right\rangle$ amplitude and increasing of the local maximum of the tangle with small oscillations (see Fig. 2b). These small oscillations will be observed even for a large interaction time. If we consider $\theta=\pi / 4$, i.e. the initial state of the Cooper pair box is given by $\rho_{A}(0)=0.5(|e\rangle\langle e|+| g\rangle\langle g|)$, we see that $\left\langle\hat{\sigma}_{z}(t)\right\rangle=0$ and the tangle tends to its maximum value. In this sense, the system has relatively high entanglement. The smallest entanglement which is almost
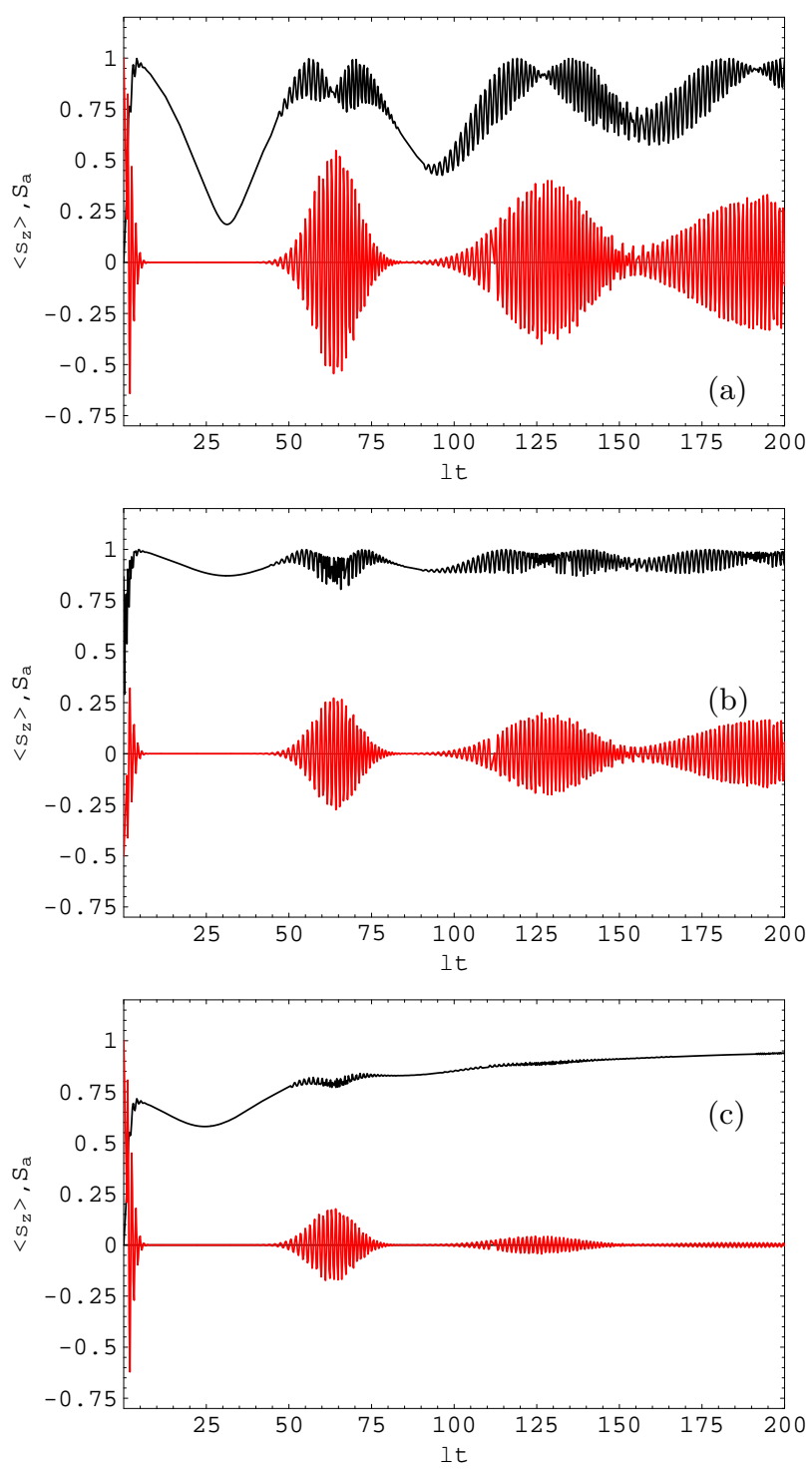

FIG. 2: (color online) Dynamics of the atomic inversion and tangle as functions of the scaled time $\lambda t$. The parameters are $\varsigma_{1}=\cos ^{2} \theta$ and $\bar{n}=25$. The other parameters are $\phi=0.1$; $\xi=\pi / 2 ; E_{J 0}=1$ and $k=1$. The three figures from above have (a) $\theta=0, \gamma=0$, (b) $\theta=\pi / 3, \gamma=0$, and (c) $\theta=0$, $\gamma=0.001$.

zero is obtained only for initial pure state case. Similarly to what we have seen in Fig. 2b, we observe how the amplitude of $\left\langle\hat{\sigma}_{z}(t)\right\rangle$ decreases as the time goes on due to the inclusion of intrinsic decoherence (see Fig. 2c). Recall that, as time increases further, the intrinsic decoherence effect leads to zero inversion and maximum entanglement.

It is interesting to mention here the fact that, in the absence of the decoherence, the final density matrix of the system Eq. (44) can be rewritten as $\hat{\rho}(t)=|\psi(t)\rangle\langle\psi(t)|$ 
where $|\psi(t)\rangle=|e\rangle \otimes\left|\Phi_{e}\right\rangle+|g\rangle \otimes\left|\Phi_{g}\right\rangle$ and $\left|\Phi_{j}\right\rangle$ are the field states 39 . From our further calculations, we show that, at a particular effective time the populations inversion and the overlap of field states vanish simultaneously. That is, the populations become equal and both the Cooper pair box and field states are mutually orthogonal. Thereby, the state equation $|\psi(t)\rangle$ becomes maximally entangled at this particular effective time. We would like to remark that the interest in studying the generation of maximally entangled states in the short time region arises due to the unavoidable presence of decoherence effects in experiments performed at the high $Q$ region due to cavity dissipation. The experimental generation of entangled states in the mesoscopic field regime has been reported for fields with an average photon number of a few tens $\underline{40}$. In this regime, after the first collapse, the field splits into two orthogonal humps in phase space. Thus, the field behaves as an effective two-level system and the entanglement of the composite system is easily described in terms of factorized states $\$ 1,42$.

\section{B. Relative entropy}

For the entangled states $\hat{\rho}(t)$ the quantum relative entropy is defined as the distance between the entangled state $\hat{\rho}(t)$ and disentangled state $\operatorname{tr}_{\mathcal{A}} \hat{\rho}(t) \otimes \operatorname{tr}_{\mathcal{B}} \hat{\rho}(t) \in$ $\mathfrak{S}\left(\mathcal{H}_{1} \otimes \mathcal{H}_{2}\right) \underline{\underline{43}}$

$$
I_{\rho}\left(\rho_{t}^{\mathcal{A}}, \rho_{t}^{\mathcal{B}}\right)=\operatorname{tr} \hat{\rho}(t)\left(\log \hat{\rho}(t)-\log \left(\operatorname{tr}_{\mathcal{A}} \hat{\rho}(t) \otimes \operatorname{tr}_{\mathcal{B}} \hat{\rho}(t)\right)\right),
$$

where $\rho_{t}^{\mathcal{A}}=\operatorname{tr}_{\mathcal{B}}(\hat{\rho}(t))$ and $\rho_{t}^{\mathcal{B}}=\operatorname{tr}_{\mathcal{A}}(\hat{\rho}(t)), \mathcal{A}(\mathcal{B})$ refers to the first (second) qubit. Note that if the entangled state $\hat{\rho}(t)$ is a pure state, $S(\hat{\rho}(t))=0$ and then $S\left(\operatorname{tr}_{\mathcal{A}} \hat{\rho}(t)\right)=S\left(\operatorname{tr}_{\mathcal{B}} \hat{\rho}(t)\right)$, which means that we have $I_{\rho}\left(\rho_{t}^{A}, \rho_{t}^{B}\right)=2 S\left(\operatorname{tr}_{\mathcal{B}} \hat{\rho}(t)\right)$.

Taking the partial trace over the junction system, we obtain $\rho_{t}^{F}=\operatorname{tr}_{A} \rho(t)$. The von Neumann entropy for the reduced state $S\left(\rho_{t}^{F}\right)$ is computed as

$$
\begin{aligned}
S\left(\rho_{t}^{F}\right)= & -\lambda_{1}^{F}(t) \log \lambda_{1}^{F}(t)-\lambda_{2}^{F}(t) \log \lambda_{2}^{F}(t) \\
& -\lambda_{3}^{F}(t) \log \lambda_{3}^{F}(t)-\lambda_{4}^{F}(t) \log \lambda_{4}^{F}(t),
\end{aligned}
$$

where $\lambda_{i}^{F}(t)$ are the solutions of the following equation $\operatorname{det}[\hat{\rho(t)}-\lambda(t) \hat{N(t)}]=0, \hat{\rho(t)}$ and $\hat{N(t)}$ are $4 \times 4$ matrices having the following elements

$$
\begin{aligned}
{[\hat{\rho(t)}]_{i j} } & \equiv\left\langle\psi_{i}(t)\left|\rho_{t}^{F}\right| \psi_{j}(t)\right\rangle, \quad(i, j=1,2,3,4), \\
{[\hat{N(t)}]_{i j} } & \equiv\left\langle\psi_{i}(t) \mid \psi_{j}(t)\right\rangle, \quad(i, j=1,2,3,4),
\end{aligned}
$$

and $\left|\psi_{j}(t)\right\rangle$ are the eigenfunctions of the following eigenvalue problem $\rho_{t}^{F}\left|\psi_{i}(t)\right\rangle=\lambda_{i}^{F}(t)\left|\psi_{i}(t)\right\rangle$.

On the other hand, the final state of the junction system is given by taking the partial trace over the field system $\rho_{t}^{J} \equiv \operatorname{tr}_{F} \rho(t)$. Then the von Neumann entropy for the reduced state $S\left(\rho_{t}^{A}\right)$ is computed by

$$
S\left(\rho_{t}^{J}\right)=-\lambda_{+}^{J}(t) \log \lambda_{+}^{J}(t)-\lambda_{-}^{J}(t) \log \lambda_{-}^{J}(t),
$$
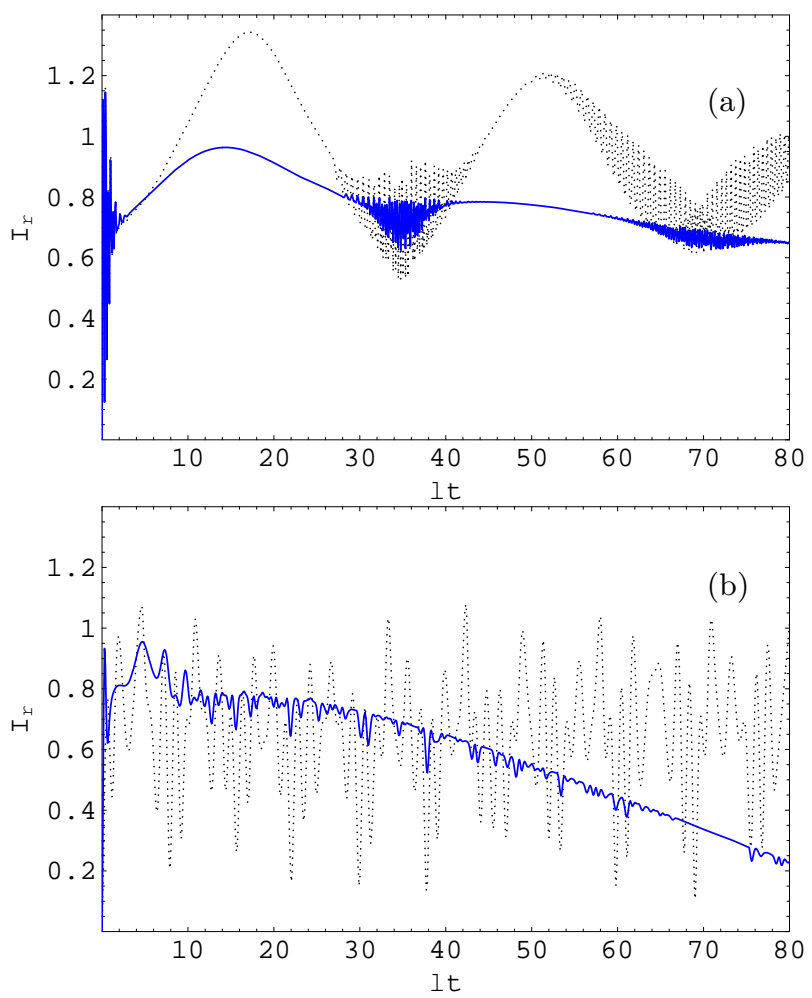

FIG. 3: (color online) Plot of $I_{\rho}\left(\rho_{t}^{\mathcal{A}}, \rho_{t}^{\mathcal{B}}\right)$ as a function of the scaled time $\lambda t$. The Cooper pair box starts from a mixed state with $\varsigma_{1}=0.9$ and the initial state of the field is a coherent state with different values of the mean photon number, where (a) $\bar{n}=30$ and (b) $\bar{n}=0.5$. The solid curve corresponds to presence of the decoherence $(\gamma=0.1 \lambda)$ and dotted curve corresponds to absence of the decoherence $(\gamma=0)$.

where $\lambda_{ \pm}^{J}(t)$ are the eigenvalues of the reduced junction state $\rho_{t}^{J}$.

Using equations (6), (7) and (9), we obtain the relative entropy, which can be used to measure the total correlation in the system under consideration. From the above equations and the matrix elements which represent the state of the field, we are able to determine under which conditions we may attain reasonable correlations between the junction and cavity field. Apart from the case represented above, in general there is no way to relate the correlation dynamics exclusively to the initial field state.

In what follows, we shall analyze numerically whether and how it would be possible to control the correlations of the output state. In typical experiments, the Cooperpair box can be made from aluminum, with an energy gap of $2.4 \mathrm{~K}$ (about $50 \mathrm{GHz}) \underline{44}$, the charge energy 149 $\mathrm{GHz}$ and the Josephson energy $13.0 \mathrm{GHz}$. The frequency of the cavity field is taken as $40 \mathrm{GHz}$, corresponding to a wavelength $\sim 0.75 \mathrm{~cm}$. As a side remark, we note that, the value $I_{\rho}\left(\rho_{t}^{\mathcal{A}}, \rho_{t}^{\mathcal{B}}\right)=2 \ln (2)$ appears in analytical criterion, based on an estimation of the composite system entanglement. It will be worth analyzing this curious co- 
incidence in order to gain a deeper understanding of the strengths and maximum points of the correlations. The points of maximum correlation can be generated either using equation (6) or, what seems more feasible, directly from Fig. (3). In this figure, we plot $I_{\rho}\left(\rho_{t}^{\mathcal{A}}, \rho_{t}^{\mathcal{B}}\right)$ as a function of the scaled time and for different values of the decoherence parameter $\gamma$, which is in agreement (at $t=0)$ with the exact calculations for the $I_{\rho}\left(\rho_{t}^{\mathcal{A}}, \rho_{t}^{\mathcal{B}}\right)$. This figure clearly shows that the correlations reaches a local maximum before the first collapse time. Returning to Fig. 3, after reaching its maximum the correlation decays exponentially as a result of the dissipation and/or the microwave field, attaining local minimum in the asymptotic limit. Furthermore, the addition of intrinsic decoherence via the parameter $\gamma$ leads to a drastic change of the correlation properties, lowering the maximum values of the total correlations. It is interesting to note that in this regime, the maximum correlations occur at a time in which the two orthogonal states are completely overlapped in phase space.

Since maximum correlation takes a finite time, it makes sense to consider whether the small values of the mean photon number can be used to change the process of correlations. It is shown that, a similar effect of the decoherence on the total correlation is seen in Fig. 3b for a small value of the mean-photon number, but the number oscillations is increased with decreasing the local minimum $I_{\rho}\left(\rho_{t}^{\mathcal{A}}, \rho_{t}^{\mathcal{B}}\right) \simeq 0.2$. We attribute our finding to the fact that in the presence of decoherence it takes some time for the junction to become entangled with the microwave field. If during this entanglement buildup the system is acted upon by an appropriately designed control, it becomes possible to channel back quantum coherence from the field to the Cooper pair box. Our results thus reinforce the superconducting charge qubits as viable candidates for quantum-information processing devices, and suggest that more refined control strategies might play an important role in future of solid-state based quantum information devices.

From the above results, one might now raise the following possibility: taking a strong decoherence regime where $\gamma \gg 0.01 \lambda$, one can estimate the exact time in which the total correlations will be vanished. Therefore, if the intrinsic decoherence is substantially strong, no correlations between the junction and the microwave field will occur, suggesting that it might be possible to obtain maximum entangled states using a weak decoherence. It is straightforward to verify, however, that in order to do so one would require a value of $\gamma$ close to zero.

\section{Concurrence}

To measure the degree of entanglement for mixed states of bipartite systems composed by two-level subsystems, one needs to consider a commonly used measure such as the concurrence $\frac{45}{\underline{4}}$ which has been proven to be a reasonable entanglement measure or negativity $\underline{46}$. Anal- ysis of the entanglement decay rates under decoherence for different models of the interaction between systems of arbitrary dimensions with the environment has been presented ${ }^{47}$. We write the standard basis of the product space of the system as $|00\rangle=|g, n\rangle,|01\rangle=|e, n\rangle,|10\rangle=$ $|g, n+1\rangle$ and $|11\rangle=|e, n+1\rangle$. For the density matrix $\hat{\rho}(t)$, which represents the state of a bipartite system, concurrence is defined as

$$
C(\hat{\rho})=\max \left\{0, \lambda_{1}-\lambda_{2}-\lambda_{3}-\lambda_{4}\right\},
$$

where the $\lambda_{i}$ are the non-negative eigenvalues, in decreasing order $\left(\lambda_{1} \geq \lambda_{2} \geq \lambda_{3} \geq \lambda_{4}\right)$, of the Hermitian matrix $\Upsilon \equiv \sqrt{\sqrt{\hat{\rho}} \widetilde{\rho} \sqrt{\hat{\rho}}}$ and $\widetilde{\rho}=\left(\sigma_{y} \otimes \sigma_{y}\right) \hat{\rho}^{*}\left(\sigma_{y} \otimes \sigma_{y}\right)$. Here, $\hat{\rho}^{*}$ represents the complex conjugate of the density matrix $\hat{\rho}$ when it is expressed in a fixed basis and $\sigma_{y}$ represents the Pauli matrix in the same basis. The function $C(\hat{\rho})$ ranges from 0 for a separable state to 1 for a maximum entanglement.

In figure (4), we plot the numerically evaluated results for the concurrence $C(\hat{\rho})$, as a function of the scaled time $\lambda t$ and phase damping parameter $\gamma$ in units of $\lambda$. The overall picture coming from Fig. (4) is that the recurrence dynamics due to large mean photon number $(\bar{n}=30)$ starts from zero, goes to a maximum and then returns to zero. In an asymptotic limit the coupled system reaches a pure state and stays in this pure state some times $(1 \leq \lambda t \leq 5.3)$ then at some finite time weak entanglement is observed. Meanwhile, as shown in Fig. (4), due to small value of the mean photon number, no entanglement at earlier times, and suddenly at a certain time entanglement starts to build up. This delayed creation of entanglement, that has been called sudden birth of entanglement $\frac{13}{}$, is opposite to the currently extensively discussed sudden death of entanglement 15 . We see that $C(\hat{\rho})$ falls off sharply as $\lambda t$ increases. The non-classical character of the field for small values of the average photon number $\bar{n}$, is reflected in larger entanglement between the junction and the microwave field. As long as the time is reasonably close to this threshold value and the mean photon number takes small values, then the delayed creation of entanglement is still obtained. However, if we start from a pure state, the asymptotic value of the concurrence can be obtained when the phase damping is increased. It is observed that, at later times decoherence destroys such structures, in agreement with Fig. 3. We have confirmed the predictions of this phenomenon using a systematic numerical analysis where a number of relevant parameters has been varied. However, once the initial state setting of the Cooper pair box is considered as a mixed state, this feature no longer exists and entanglement vanishes in an asymptotic limit (see Fig. (4).

\section{PHASE SPACE APPROACH}

To give a more detailed discussion, we are now going to focus our attention on the field dynamics. The representation of fields in phase space has been providing 

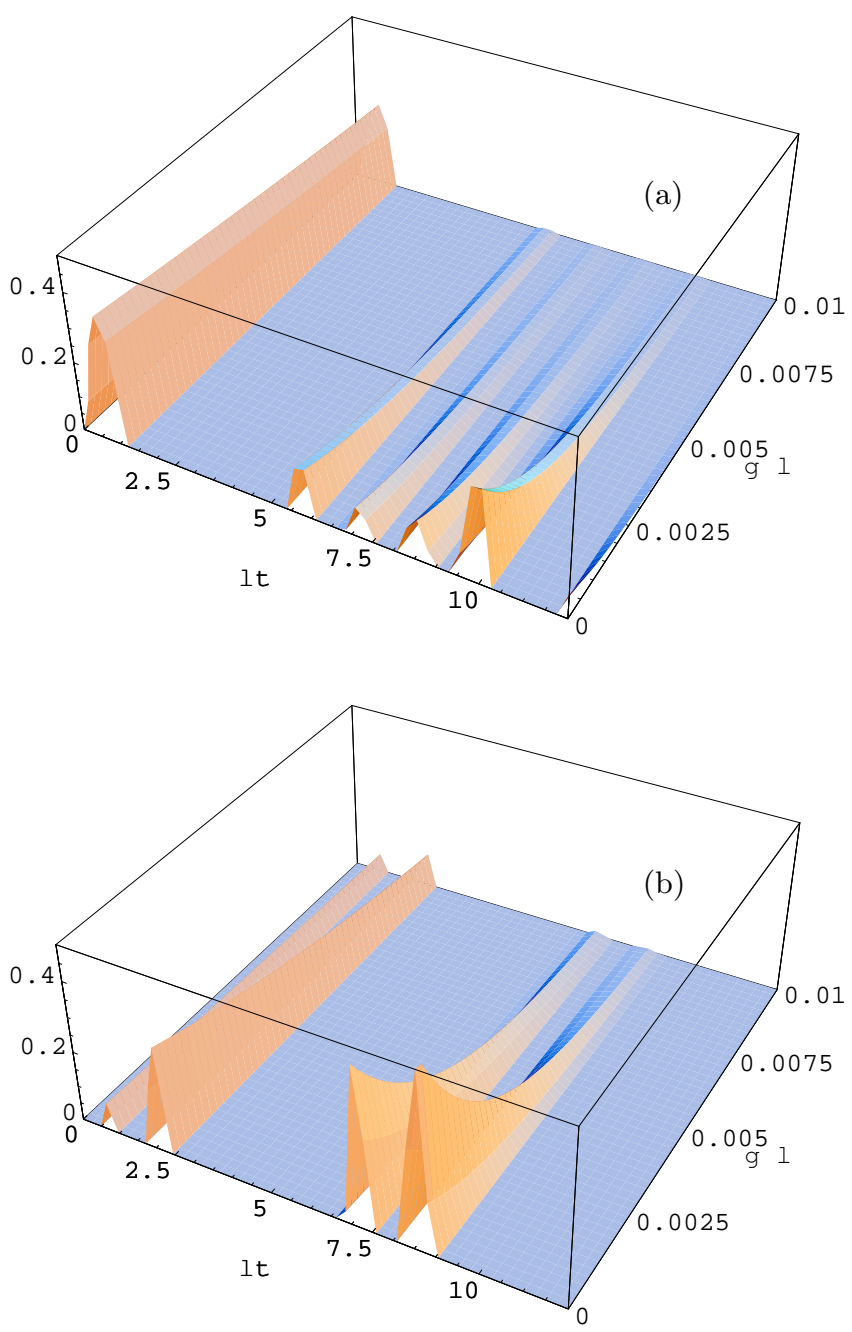

FIG. 4: (color online) Development of the concurrence $C(\rho)$ as a function of the scaled time $\lambda t$ and the decoherence parameter $\gamma / \lambda$. The other parameters are the same as figure 3.

new insights of the two-level system dynamics 48 . Perhaps the most convenient quasiprobability to be used in this kind of problem is the $W$-function. In this connection, it seems interesting to consider the Wigner function corresponding to the final state of the present system. Experimentally these functions can be measured via homodyne tomography $\underline{49}$. Since the quasi-probability distribution function is the Fourier transform of the characteristic function, therefore the Wigner function can be obtained from the direct evaluation of the integral

$$
W(x, p)=\frac{1}{\pi} \int_{-\infty}^{\infty}\left\langle x-x^{\prime}|\rho(t)| x+x^{\prime}\right\rangle \exp \left(2 i p x^{\prime}\right) d x^{\prime},
$$

According to Eq. (11) we have plotted in Fig. 5, the Wigner function $W(x, p)$ against the parameters $x$ and
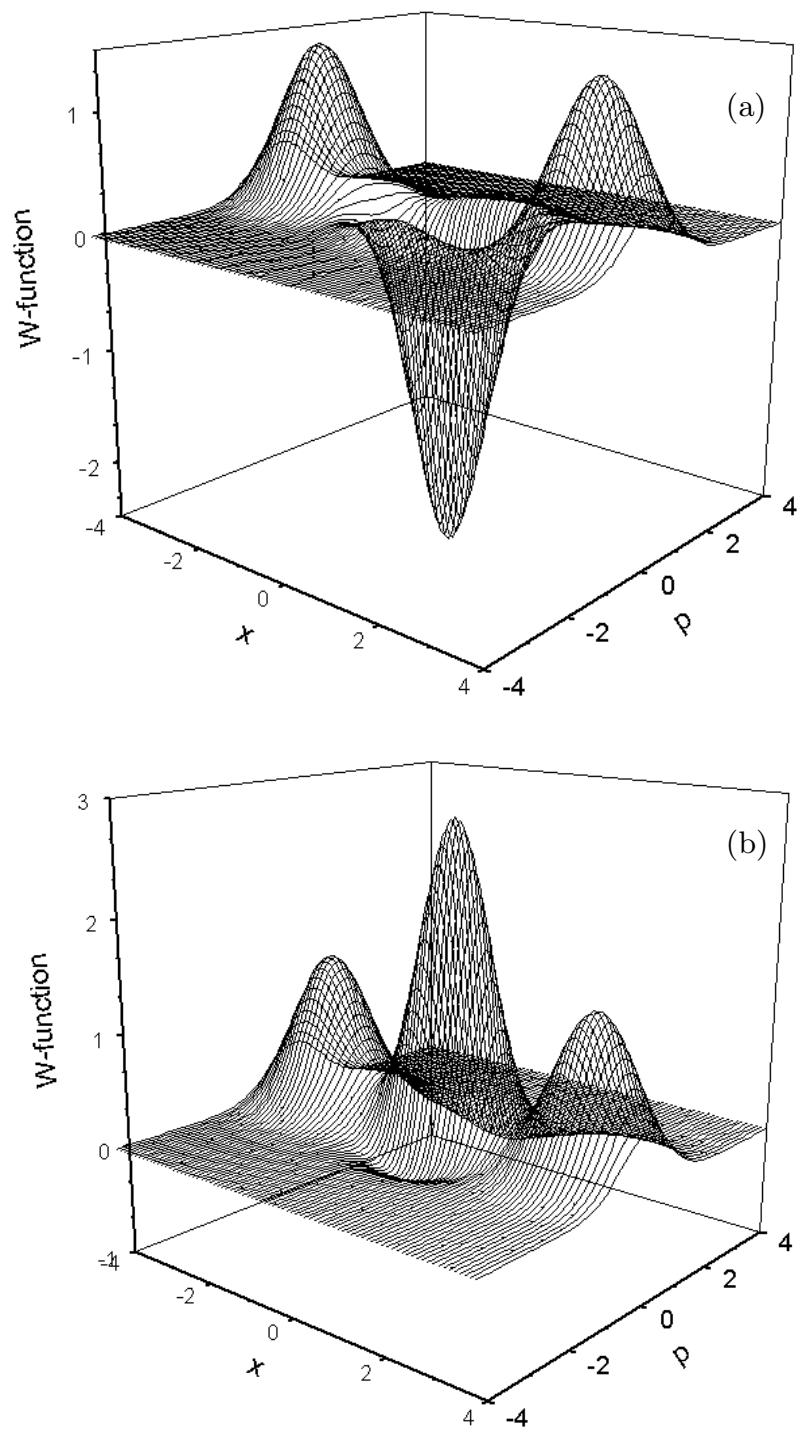

FIG. 5: Wigner distribution of the cavity field as function of $x$ and $p$. The parameters are $\alpha=2.1, t=0, \theta=\pi / 2$, (b) $\alpha=$ $2.1, t=0, \theta=0$. In this case we have assumed the initial state of the junction is given by $|\psi(0)\rangle_{J}=\cos (\theta / 2)|e\rangle+\sin (\theta / 2)|g\rangle$.

$p$ for the initial state of the filed as coherent state. If the Cooper pair box starts from a superposition state $(\theta=\pi / 2)$, we see that the Wigner function has negative values with a very little structure around the base, see Fig. 11 $\mathrm{k}$. This in fact is a signature of the nonclassical effect. These negative values will not be seen if the Cooper pair box starts from a pure state Fig. 11b. From our further calculations, one may see that due to the decoherence the quasiprobability distribution function is not as negative as in absence of the decoherence. Of course, for greater values of the decadence parameter the effect would be stronger. For $t>0$ the two peaks split into two sets of counter-rotating peaks during the collapse. At longer times the $W$-function is spread out 
over an angular region in the $x y$-plane. If we combine this observation with the fact that the entanglement degree at this moment is almost maximum we can conclude that the cavity field is in a pure state. As shown in 48 although decoherence destroys the quantumness of the field, information of the initial field may be obtained via the reconstruction of quasiprobability distribution functions.

\section{CONCLUDING REMARKS}

We have extended the exactly solvable model of a single-Cooper-pair box with a nonclassical microwave field, taking into account the decoherence effect. We considered the charge-qubit with a superconductingquantum-interference-device loop and used the microwave field to change the flux through the loop. This treatment puts the so far phenomenological description of charge-qubit systems on a firm footing and paves the way for a variety of future applications in particular because the extension to an arbitrary number of charge qubits is straightforward. Collapse and revival phenomenon have been predicted via the Josephson effect that involve interactions of the photons with the junction. The revivals which may be seen in population inversion for small values of the decoherence parameter will not be occurred for stronger decoherence. It is shown that, for certain values of the initial average photon number, maximally entangled state is generated.

We have presented a detailed analysis of the dynamics of the process of the total correlation in a junction-field system. For the weak-field region, the behavior is essentially oscillatory, while for the strong-field region, the correlation tends to be randomized in the evolution, although showing collapses and revivals. To measure the degree of entanglement for mixed states of junction-field system, we have considered the concurrence which is a commonly used measure. We have investigated numerically results of the concurrence by considering the influences of the mean-photon number and intrinsic decoherence in the junction-field system. We have shown an interesting phenomenon of delayed birth of entanglement that initially separable junction and field become entangled after a finite time. Similar to the correlation case, the decoherence leads to lowering the maximum entanglement and further increase of the decoherence leads to entanglement sudden death. Further analysis using the evolution of the field $W$-function showed us that for a given field coherent intensity $\bar{n}$, there is an optimum value of the decoherence parameters for which the quasiprobability distribution has no negative values. Given the general interest in superconducting qubits as a device for quantum information processing, including the realization of complex single-qubit manipulation schemes and the generation of entangled states 50 , we feel these results may find great utility in future applications.

\section{Acknowledgments}

We gratefully acknowledge Jason Ralph and M A Bouchene for truly enlightening discussions and useful criticism of this work. MA gratefully acknowledges the financial support from Universit Paul Sabatier.
* Electronic address: abdelaty@hotmail.com

$\dagger$ Electronic address: m.j.everitt@physics.org

1 J. Q. You and F. Nori, Phys. Today 58, 42 (2005).

2 Y. Makhlin, G. Schön, and A. Shnirman, Rev. Mod. Phys. 73, 357 (2001).

3 D.V. Averin, Fortschrit. der Physik 48, 1055 (2000).

4 J.M. Martinis, S. Nam, J. Aumentado, and C. Urbina, Phys. Rev. Lett. 89, 117901 (2002).

${ }^{5}$ F. Benatti, R. Floreanini and J. Realpe-Gomez, J. Phys. A: Math. Theor. 41, 235304 (2008)

6 J. Q. You and F. Nori, Phys. Rev. B 68, 064509 (2003).

7 V. Vedral, M. B. Plenio and P. L. Knight, "The Physics of Quantum Information", edited by D Bouwmeester, A Ekert and A Zeilinger, Springer (2000); V. Vedral, M. B. Plenio, K. Jacobs, and P. L. Knight, Phys. Rev. A 56, 4452 (1997).

8 A. Al-Qasimi and D. F. V. James, Phys. Rev. A 77, 012117 (2008).

9 G.J. Milburn, Phys. Rev. A 44, 5401 (1991)

10 M.J. Everitt, W.J. Munro and T.P. Spiller, Phys. Rev. A 79, 032328 (2009).

11 B. Bellomo, R. L. Franco and G. Compagno, Phys. Rev. Lett. 99, 160502 (2007);

B. Bellomo, R. L. Franco and G. Compagno, Phys. Rev.
A 77, $032342(2008)$;

J. Dajka, M. Mierzejewski and J. Luczka, Phys. Rev. A 77, 042316 (2008);

X. Cao and H. Zheng, Phys. Rev. A 77, 022320 (2008).

12 M. Ikram, F. L. Li and M. S. Zubairy, Phys. Rev. A 75, 062336 (2007).

13 Z. Ficek and R. Tanas, Phys. Rev. A 74, 024304 (2006).

14 T. Konrad, F. de Melo, M. Tiersch, C. Kasztelan, A. Aragao and A. Buchleitner, Nature Physics 4, 99 (2008)

15 T. Yu and J.H. Eberly, Science 316, 555 (2007)

16 Y. Makhlin, G. Schön and A. Schnirman, Rev. Mod. Phys. 73, 357 (2001); E. Zipper, M. Kurpas, M. Szelg, J. Dajka and M. Szopa, Phys. Rev. B 74, 125426 (2006)

17 Y. Liu, L. F. Wei, and F. Nori1, Phys. Rev. A 71, 063820 (2005)

18 M. Steffen, M. Ansmann, R. C. Bialczak, N. Katz, E. Lucero, R. McDermott, M. Neeley, E. M. Weig, A. N. Cleland, and J. M. Martinis, Science, 5792, 1423 (2006); Yu. A. Pashkin et al. Int. J. Quant. Info. 1, 421 (2003)

19 R. Migliore, A. Messina and A. Napoli, Eur. Phys. J. B 13, 585 (2000); 22, 111 (2001)

${ }^{20}$ W. Krech and T. Wagner, Phys. Lett. A 275, 159 (2000); M. Zhang, J. Zpu and B. Shao, Int. J. Mod. Phys. B 16, 4767 (2002) 
${ }^{21}$ E. T. Jaynes and F. W. Cummings, Proc. IEEE 51, 89 (1963).

22 C. C. Gerry and P. L. Knight, Introductory Quantum Optics, chapter 4 (Cambridge University Press, 2005).

23 J. H. Eberly, N. B. Narozhny and J. J. SanchezMondragon, Phys. Rev. Lett. 44, 1323 (1980).

24 N. B. Narozhny, J. J. Sanchez-Mondragon and J. H. Eberly, Phys. Rev. A 23, 236 (1981).

25 P. L. Knight and P. M. Radmore, Phys. Rev. A 26, 676 (1982).

26 J. Gea-Banacloche, Phys. Rev. Lett. 65, 3385 (1990).

27 J. Gea-Banacloche, Phys. Rev. A 44, 5913 (1991).

28 V. Bužek and P. L. Knight, Prog. Opt. 34, 1 (1995).

40 A. Auffeves, P. Maioli, T. Meunier, S. Gleyzes, G. Nogues, M. Brune, J. M. Raimond and S. Haroche, Phys. Rev. Lett. 91, 230405 (2003).

30 T. Meunier, S. Gleyzes, P. Maioli, A. Auffeves, G. Nogues, M. Brune, J. M. Raimond and S. Haroche, Phys. Rev. Lett. 94, 010401 (2005).

31 Schneider and G.J. Milburn, Phys. Rev. A 57, 3748 (1998); S. Schneider and G.J. Milburn, Phys. Rev. A 59, 3766 (1999).

32 Liu Xiao-Nan and Shao Bin and Zou Jian, Chin. Phys. Lett. 222997 (2005); Qiu Liang, Wang An-Min and Ma Xiao-San, Commun. Theor. Phys. 49516 (2008); S Shelly Sharma, N Sharma, Journal of Optics B 7230 (2005); K Kimm and H Kwon, Phys. Rev. A 65022311 (2002).

${ }^{33}$ H.-P. Breuer and F. Petruccione, The theory of open quantum systems, Oxford University Press, Oxford, (2002)

34 D. A. Lidar and K. B. Whaley, in Irreversible quantum dynamics edited F.Benatti and R. Floreanini, Spring Lecutre Notes in Physics, Vol. 62, Berlin (2003), p.83.

${ }^{35}$ C. W. Gardiner and P. Zoller, Quantum Noise (SpringerVerlag, Berlin, 2000);M. P. Almeida, F. de Melo, M. Hor-
Meyll, A. Salles, S. P. Walborn, P. H. Souto Ribeiro, and L. Davidorich, Science 316, 579 (2007).

36 P. Rungta, V. Bužek, C. M. Caves, H. Hillery, and G. J. Milburn, Phys. Rev. A 64, 042315 (2001).

37 J. F. Ralph, E. J. Grifth, T. D. Clark and M. J. Everitt, Phys. Rev. B 70, 214521 (2004)

38 P. I. Knight and P. M. Radmore, Phys. Lett. A 90, 342 (1982)

39 C. C. Gerry and P. L. Knight, Introductory Quantum Optics (Cambridge University Press, Cambridge, 2005).

40 A. Auffeves, P. Maioli, T. Meunier, S. Gleyzes, G. Nogues, M. Brune, J.M. Raimond, S. Haroche, Phys. Rev. Lett. 91, 230405 (2003).

41 J. Gea-Banacloche, T.C. Burt, P.R. Rice, L.A. Orozco, Phys. Rev. Lett. 94, 053603 (2005).

42 J.C. Retamal, A. Delgado, C. Saavedr, Phys. Lett. A 355, 7 (2006)

43 V. Vedral and M. B. Plenio, Phys. Rev. A 57, 1619 (1998); M. Abdel-Aty, S. Furuichi and A.-S. F. Obada, J. Opt. B: Quant. Semiclass. Opt. 4, 37 (2002); S. Furuichi and M. Abdel-Aty, J. Phys. A: Math. 6 Gen. 34, 6851 (2001).

${ }^{44}$ K.W. Lehnert, K. Bladh, L.F. Spietz, D. Gunnarsson, D.I. Schuster, P. Delsing, and R.J. Schoelkopf, Phys. Rev. Lett. 90, 027002 (2003); Yu-xi Liu, L.F. Wei, and F. Nori, Phys. Rev. A 72, 033818 (2005)

45 F. Mintert, A. R. R. Carvalhoa, M. Kus, A. Buchleitner, Phys. Rep. 415, 207 (2005)

46 G. Vidal and R. Werner, Phys. Rev. A 65, 032314 (2002).

47 A. R. R. Carvalho, F. Mintert, S. Palzer, and A. Buchleitner, Eur. Phys. J. D 41, 425 (2007)

48 H. Moya-Cessaa, Phys. Rep. 432, 1 (2006)

49 B. Yurke, D. Stoler, Phys. Rev. Lett. 57, 3 (1986).

50 T. Yamamoto et al., Nature 425, 941 (2003). 